INTERVENTIONAL CARDIOLOGY AND SURGERY

\title{
Socioeconomic deprivation is a predictor of poor postoperative cardiovascular outcomes in patients undergoing coronary artery bypass grafting
}

\author{
F C Taylor, R Ascione, K Rees, P Narayan, G D Angelini
}

Heart 2003;89: 1062-1066

See end of article for authors' affiliations

Correspondence to: Dr R Ascione, Bristol Heart Institute, University of Bristol, Bristol Royal Infirmary, Bristol BS2 8HW, UK; r.ascione@bristol.ac.uk

Accepted 13 March 2003

\begin{abstract}
Objective: To investigate the effects of socioeconomic deprivation on cardiovascular risk factors and postoperative clinical outcomes of patients undergoing coronary artery bypass grafting (CABG).

Design: Retrospective analysis of prospectively collected data.

Setting: Surgical population of the southwest of England, April 1996 and August 2000.

Study group: Data on 3578 consecutive patients undergoing CABG at the Bristol Royal Infirmary NHS Trust were abstracted. Data were retrieved from the Patient Analysis \& Tracking System. Carstairs index was used to measure socioeconomic deprivation of area of residence and was divided into five quintiles, where quintile 1 denotes least deprived and 5 most deprived.

Outcome measures: End points were postoperative complications and 30 day mortality.

Results: Higher deprivation scores were associated with younger age $(p<0.004)$, greater body mass index, diabetes, smoking at time of surgery, and higher EuroSCOREs (all $p \leqslant 0.001$ ). After adjustment for EuroSCORE, socioeconomic deprivation was independently associated with postoperative myocardial infarction $(p=0.05)$ and combined postoperative myocardial infarction, stroke, and death $(p=0.016)$. Hospital length of stay for the patients in the highest quintiles was also significantly longer than for those in the lower quintiles $(p=0.04)$.

Conclusion: Patients undergoing CABG living in areas with high deprivation scores are younger, have more clinical risk factors, and experience more postoperative cardiovascular complications than patients living in low deprivation score areas.
\end{abstract}

S ocioeconomic deprivation is associated with morbidity and mortality from coronary artery disease, ${ }^{12}$ reduced access to specialist cardiac services, ${ }^{3}$ less uptake of non-invasive cardiac investigations ${ }^{4}$ and revascularisation procedures, ${ }^{15-8}$ and uptake of cardiac rehabilitation. ${ }^{9}$ This is an important issue for the current health care agenda since the magnitude of socioeconomic differentials in mortality from cardiovascular disease has increased over the past decades in the UK and in several European countries. ${ }^{10}{ }^{11}$ In recent decades coronary artery bypass grafting (CABG) as a result of continuous improvement in quality of care has been established as an effective treatment in the advanced stages of coronary artery disease. ${ }^{12}{ }^{13}$ However, the influence of socioeconomic deprivation on early inhospital outcomes in patients undergoing $\mathrm{CABG}$ remains controversial.

The availability of a database to collect clinical details prospectively of all patients at our institution provides an opportunity to investigate the association between socioeconomic deprivation, preoperative risk factors, postoperative complications, and 30 day post-CABG mortality in one cardiac centre in the southwest of England.

\section{METHODS}

\section{Patient selection and data collection}

The study site covers a population of one million, with approximately $40 \%$ of patients referred from other health authorities throughout the southwest of England. We undertook a retrospective evaluation of all patients undergoing CABG at our institution from April 1996 and August 2000. A standard set of perioperative clinical data were collected prospectively for all patients. Data were entered on each patient prospectively by anaesthetists, surgeons, staff within the intensive care unit and high dependency unit, and ward nurses on to the Patient Analysis \& Tracking System (Dendrite Clinical Systems, London, UK). Data were patient demographics, cardiovascular risk factors (body mass index, hypertension, cholesterol, diabetes, previous myocardial infarction, and smoking at the time of surgery), the EuroSCORE, which assesses preoperative risk (including variables such as sex, age, previous myocardial infarction, left ventricular function, angina class, endocarditis), ${ }^{14}$ and perioperative clinical outcomes. Area deprivation scores have been used for many purposes, particularly analysis for health care services. The Carstairs index for the 1981 census of Scotland, developed by Vera Carstairs and Russell Morris, is considered a benchmark index. ${ }^{15}$ This is based on 1991 census data on car ownership, unemployment, overcrowding, and social class within postcodes. Initially, scores were arbitrarily divided into seven categories for 1981. The division into seven groups was maintained in 1991 simply to have a similar number of groups containing the same proportions of the whole population for comparison purpose. Others have used four categories for a much smaller population.

The Carstairs index, derived from patients' postcodes, was also used to measure socioeconomic deprivation in our study. The entire study population was equally divided into five quintiles (where quintile 1 denotes least deprived and 5 most deprived) to provide the best power to identify trends.

\section{Clinical management}

Anaesthetic and surgical techniques were standardised throughout the study period as previously reported. ${ }^{16}$ At the end of surgery, patients were transferred to the intensive care unit and managed in accordance with unit protocol. ${ }^{16}$ Forced air warming was used until a stable nasopharyngeal temperature of $37^{\circ} \mathrm{C}$ was reached. Patients were extubated as soon as 
Table 1 Preoperative data: Carstairs deprivation index

\begin{tabular}{|c|c|c|c|c|c|c|}
\hline Patient characteristics & $\begin{array}{l}\text { Quintile } 1 \text { median } \\
-3.55(n=719)\end{array}$ & $\begin{array}{l}\text { Quintile } 2 \text { median } \\
-2.64(\mathrm{n}=700)\end{array}$ & $\begin{array}{l}\text { Quintile } 3 \text { median } \\
-1.71(\mathrm{n}=711)\end{array}$ & $\begin{array}{l}\text { Quintile } 4 \text { median } \\
-0.63(n=712)\end{array}$ & $\begin{array}{l}\text { Quintile } 5 \text { median } \\
1.22(n=705)\end{array}$ & $\mathrm{p}$ Value \\
\hline Sex (male) & $594(83 \%)$ & $582(83 \%)$ & $553(78 \%)$ & $570(80 \%)$ & $563(79 \%)$ & 0.62 \\
\hline Age (years) & $63.3(8.7)$ & $63.1^{\prime}(8.6)$ & $63.6(8.66)$ & $63.2(9.0 \%)$ & $61.9(9.5 \%)$ & 0.004 \\
\hline Time on waiting list (days) & 122.5 (139.1) & $132.3(162.3)$ & $136.8(157.6)$ & 128.5 (144.8) & $136.2(155.0)$ & 0.33 \\
\hline \multicolumn{7}{|l|}{ Risk factors } \\
\hline Body mass index & $26.6(4.0)$ & $26.8(3.6)$ & $26.8(3.8)$ & $27.0(3.9)$ & $27.7(4.1)$ & $<0.001$ \\
\hline Diabetes & $90(12.5 \%)$ & $95(13.6 \%)$ & $106(15.9 \%)$ & $118(16.6)$ & $138(19.6 \%)$ & $<0.001$ \\
\hline Smoking at time of surgery & $508(70.7 \%)$ & $501(71.5 \%)$ & 507 (71.3\%) & $557(77.9 \%)$ & $576(81.7 \%)$ & $<0.001$ \\
\hline Cholesterol (>5 mmol/l) & $518(72.2 \%)$ & 495 (70.7\%) & 495 (69.6\%) & $483(67.8 \%)$ & $483(68.5 \%)$ & 0.05 \\
\hline Hypertension & $399(55.5 \%)$ & $406(58.1 \%)$ & $415(58.4 \%)$ & $408(57.3 \%)$ & $381(54.0 \%)$ & 0.54 \\
\hline Previous myocardial infarction & $310(43.2 \%)$ & $309(44.1 \%)$ & $324(45.6 \%)$ & $326(45.8 \%)$ & $314(44.5 \%)$ & 0.43 \\
\hline \multicolumn{7}{|l|}{ Extent of coronary artery disease } \\
\hline Single vessel & $58(8 \%)$ & $51(7 \%)$ & $47(7 \%)$ & 48 (7\%) & $47(7 \%)$ & 0.73 \\
\hline Double vessel & $167(23 \%)$ & $160(23 \%)$ & $154(22 \%)$ & $159(22 \%)$ & $182(25 \%)$ & \\
\hline Triple vessel & $475(67 \%)$ & $474(67 \%)$ & $489(69 \%)$ & $495(69 \%)$ & $470(67 \%)$ & \\
\hline Missing information & $19(2 \%)$ & $15(3 \%)$ & $21(2 \%)$ & $10(2 \%)$ & $6(1 \%)$ & \\
\hline \multicolumn{7}{|l|}{ Operative priority } \\
\hline Elective & $419(58 \%)$ & $410(58 \%)$ & $429(60 \%)$ & $435(61 \%)$ & $509(58 \%)$ & 0.94 \\
\hline Urgent & $286(39 \%)$ & $277(39 \%)$ & $268(37 \%)$ & $262(37 \%)$ & $279(39 \%)$ & \\
\hline Emergency & $11(2 \%)$ & $12(2 \%)$ & $10(2 \%)$ & $13(2 \%)$ & $12(2 \%)$ & \\
\hline Salvage & $3(1 \%)$ & $1(1 \%)$ & $1(1 \%)$ & $2(1 \%)$ & $3(1 \%)$ & \\
\hline EuroSCORE & $0.43(1.2)$ & $0.48(1.2)$ & $0.56(1.4)$ & $0.59(1.4)$ & $0.63(1.5)$ & 0.03 \\
\hline
\end{tabular}

they met the following criteria: haemodynamic stability, no excessive bleeding ( $<80 \mathrm{ml} /$ hour), normothermia, and consciousness with pain control. Fluid management postoperatively consisted of $5 \%$ dextrose infused at $1 \mathrm{ml} / \mathrm{kg} / \mathrm{h}$, with additional colloid or blood to maintain normovolemia and haematocrit greater than $24 \%$. Potassium and magnesium deficiencies were promptly treated as necessary to maintain electrolyte balance within the normal range.

\section{Clinical data, monitoring and definitions}

Mortality was defined as any death that occurred within 30 days of operation either in hospital or in the community. Clinical diagnostic criteria for postoperative myocardial infarction were new $\mathrm{Q}$ waves $>0.04 \mathrm{~ms}$ or a reduction in $\mathrm{R}$ waves $>25 \%$ in at least two leads. ST elevation was considered significant if $>1 \mathrm{~mm}$. Pacing, arrhythmias, and inotropic requirement were also recorded and defined as previously

Table 2 Perioperative data

\begin{tabular}{|c|c|c|c|c|c|c|}
\hline Outcome & $\begin{array}{l}\text { Quintile } 1 \\
(n=719)\end{array}$ & $\begin{array}{l}\text { Quintile } 2 \\
(n=700)\end{array}$ & $\begin{array}{l}\text { Quintile } 3 \\
(n=711)\end{array}$ & $\begin{array}{l}\text { Quintile } 4 \\
(n=712)\end{array}$ & $\begin{array}{l}\text { Quintile } 5 \\
(n=705)\end{array}$ & $\mathrm{p}$ Value \\
\hline \multicolumn{7}{|l|}{ Inotrope use } \\
\hline None & $508(71 \%)$ & $468(67 \%)$ & $466(66 \%)$ & $498(69 \%)$ & $430(61 \%)$ & \multirow[t]{3}{*}{0.004} \\
\hline Modest & $157(22 \%)$ & $181(26 \%)$ & $193(27 \%)$ & $158(22 \%)$ & 205 (29\%) & \\
\hline Significant & $46(6 \%)$ & $44(6 \%)$ & $44(6 \%)$ & $50(8 \%)$ & $62(9 \%)$ & \\
\hline Blood loss (ml) & $1007.4(572.0)$ & $977.9(530.0)$ & 968.7 (545.7) & $939.6(475.2)$ & $924.4(519.9)$ & 0.08 \\
\hline \multicolumn{7}{|l|}{ ST segment $>1 \mathrm{~mm}$} \\
\hline None & $619(86 \%)$ & $621(89 \%)$ & $613(86 \%)$ & $611(86 \%)$ & $602(85 \%)$ & \multirow[t]{4}{*}{0.56} \\
\hline Before CABG & $28(4 \%)$ & $28(4 \%)$ & $36(5 \%)$ & $39(5 \%)$ & $36(5 \%)$ & \\
\hline After CABG & $39(5 \%)$ & $28(4 \%)$ & $35(5 \%)$ & $28(4 \%)$ & $38(5 \%)$ & \\
\hline Before and after $C A B G$ & $25(4 \%)$ & $17(2 \%)$ & $18(3 \%)$ & $28(4 \%)$ & $21(4 \%)$ & \\
\hline \multicolumn{7}{|l|}{ Arrhythmias } \\
\hline None & 607 (84\%) & $604(86 \%)$ & $605(85 \%)$ & $598(84 \%)$ & $592(85 \%)$ & \multirow[t]{5}{*}{0.93} \\
\hline SVT & $13(2 \%)$ & $12(2 \%)$ & $16(2 \%)$ & $16(2 \%)$ & $12(1 \%)$ & \\
\hline Any pacing & $81(11 \%)$ & $76(10 \%)$ & $79(9 \%)$ & $81(11 \%)$ & $87(12 \%)$ & \\
\hline VF/VT & $13(2 \%)$ & $7(1 \%)$ & $9(2 \%)$ & $12(2 \%)$ & $12(1 \%)$ & \\
\hline Missing data & $5(1 \%)$ & $1(1 \%)$ & $2(1 \%)$ & $5(1 \%)$ & $2(1 \%)$ & \\
\hline \multicolumn{7}{|l|}{ Pulmonary complications } \\
\hline None & $632(87 \%)$ & $623(89 \%)$ & $635(89 \%)$ & $625(88 \%)$ & $602(85 \%)$ & \multirow[t]{4}{*}{0.21} \\
\hline Yes & $78(10 \%)$ & $70(8 \%)$ & $71(9 \%)$ & $76(9 \%)$ & $95(13 \%)$ & \\
\hline Mini tracheostomy & $3(1 \%)$ & $2(1 \%)$ & $2(1 \%)$ & $1(1 \%)$ & $3(1 \%)$ & \\
\hline Full tracheostomy & $5(1 \%)$ & $0(1 \%)$ & $3(1 \%)$ & $8(1 \%)$ & $5(1 \%)$ & \\
\hline \multicolumn{7}{|l|}{ Renal complications } \\
\hline None & $681(95 \%)$ & $666(95 \%)$ & $670(94 \%)$ & $675(95 \%)$ & $664(94 \%)$ & \multirow[t]{4}{*}{0.83} \\
\hline Rise in creatinine $>200 \mathrm{mmol} / /$ & $26(4 \%)$ & $23(3 \%)$ & $28(3 \%)$ & $25(3 \%)$ & $26(4 \%)$ & \\
\hline HF dialysis required & $12(1 \%)$ & $7(1 \%)$ & $13(1 \%)$ & $11(1 \%)$ & $13(1 \%)$ & \\
\hline Missing data & $0(0 \%)$ & $4(1 \%)$ & $0(0 \%)$ & $1(1 \%)$ & $2(1 \%)$ & \\
\hline \multicolumn{7}{|l|}{ Neurological complications } \\
\hline None & 705 (98\%) & $683(96 \%)$ & $698(97 \%)$ & $687(96 \%)$ & $682(97 \%)$ & \multirow[t]{4}{*}{0.36} \\
\hline GCS $<15$ & $9(1 \%)$ & $9(1 \%)$ & $6(1 \%)$ & $12(1 \%)$ & $8(1 \%)$ & \\
\hline Peripheral nerve damage & $0(0 \%)$ & $2(1 \%)$ & $1(1 \%)$ & $1(1 \%)$ & $1(1 \%)$ & \\
\hline TIA/stroke & $5(1 \%)$ & $3(1 \%)$ & $6(1 \%)$ & $9(1 \%)$ & $14(1 \%)$ & \\
\hline
\end{tabular}

${ }^{*} \chi^{2}$ test for trend across the groups.

Variables are reported as frequencies (percentage) or where applicable means (SD).

CABG, coronary artery bypass grafting; GCS, Glasgow coma score; HF, haemofiltration; SVT, supraventricular tachyarrhythmias; TIA, transient ischaemic attack; VF, ventricular fibrillation; VT, ventricular tachycardia. 
Table 3 Postoperative data adjusted for EuroSCORE: Carstairs deprivation score, reported as odds ratios and $95 \%$ confidence intervals (CI) adjusted for EuroSCORE

\begin{tabular}{|c|c|c|c|c|c|c|}
\hline & $\begin{array}{l}\text { Quintile } 1 \\
(\mathrm{n}=719)\end{array}$ & $\begin{array}{l}\text { Quintile } 2 \\
(\mathrm{n}=700)\end{array}$ & $\begin{array}{l}\text { Quintile } 3 \\
(n=711)\end{array}$ & $\begin{array}{l}\text { Quintile } 4 \\
(n=712)\end{array}$ & $\begin{array}{l}\text { Quintile } 5 \\
(\mathrm{n}=705)\end{array}$ & $\chi^{2}(4 \mathrm{df})$ \\
\hline 30 day mortality after $C A B G$ & $9(1.2 \%)$ & $6(0.8 \%)$ & $6(0.8 \%)$ & $10(1.4 \%)$ & $10(1.4 \%)$ & $0.81(1.57)$ \\
\hline Odds ratio & 1.30 & 0.75 & 0.72 & 1.17 & 1.16 & \\
\hline $95 \% \mathrm{Cl}$ & 1.13 to 1.50 & 0.26 to 2.20 & 0.24 to 2.09 & 0.46 to 3.17 & 0.45 to 2.29 & \\
\hline Postoperative myocardial infarction & $13(1.8 \%)$ & $7(1.0 \%)$ & $16(2.2 \%)$ & $13(1.8 \%)$ & $23(3.3 \%)$ & $0.05(9.26)$ \\
\hline Odds ratio & 1.03 & 0.59 & 1.34 & 1.09 & 1.96 & \\
\hline $95 \% \mathrm{Cl}$ & 0.87 to 1.21 & 0.23 to 1.51 & 0.63 to 2.68 & 0.49 to 2.41 & 0.96 to 3.98 & \\
\hline Postoperative stroke & $4(1 \%)$ & $2(1 \%)$ & $4(1 \%)$ & $5(1 \%)$ & $5(1 \%)$ & $0.64(2.50)$ \\
\hline Odds ratio & 0.80 & 0.68 & 1.37 & 1.73 & 2.10 & \\
\hline $95 \% \mathrm{Cl}$ & 0.49 to 1.31 & 0.11 to 4.13 & 0.30 to 6.16 & 0.41 to 7.29 & 0.52 to 8.44 & \\
\hline $\begin{array}{l}\text { Death, postoperative stroke, and } \\
\text { myocardial infarction }\end{array}$ & $26(3 \%)$ & $15(2 \%)$ & $26(3 \%)$ & $22(3 \%)$ & $35(5 \%)$ & $0.016(12.17)$ \\
\hline Odds ratio & 1.14 & 0.48 & 1.12 & 0.94 & 1.52 & \\
\hline $95 \% \mathrm{Cl}$ & 1.02 to 1.27 & 0.23 to 0.99 & 0.63 to 1.99 & 0.52 to 1.71 & 0.88 to 2.60 & \\
\hline Length of stay after CABG (days) * & $7.3(3.7)$ & $7.4(3.5)$ & $7.7(5.1)$ & $7.7(4.8)$ & $8.0(5.8)$ & 0.03 \\
\hline
\end{tabular}

reported..$^{15}$ Pulmonary complications were chest infection, ventilation failure, reintubation, and tracheostomy. Postoperative blood loss was defined as total chest tube drainage. Neurological complications were permanent and transient stroke. Renal complications were acute renal failure as defined by the requirement for haemodialysis. Finally, infective complications were septicaemia, and sternal and leg wound infection as defined by positive culture and requiring antibiotics. ${ }^{16}$

\section{Statistical analysis}

Following the method of Carstairs and Morris, ${ }^{15}$ each variable (that is, car ownership, unemployment, overcrowding, and social class) was standardised to have a population weighted mean of zero and a variance of one to allow each variable to have an equal influence on the resultant score. Standardisation was achieved by subtracting the all population mean from each variable and dividing the result by the standard deviation of that variable. This is called the $\mathrm{z}$ score method and the sum of these new standardised variables produces the deprivation score for each postcode sector.

Differences in patient baseline risk factors and clinical outcomes were analysed across the five quintiles using the $\chi^{2}$ test (trend) for categorical variables and analysis of variance for continuous variables. Means and standard deviations are reported for continuous variables. Significance was taken at $p \leqslant 0.05$ (two sided). Logistic regression analysis was used to examine the association of Carstairs index with clinical outcomes adjusted EuroSCORE using Stata statistical software (Stata Corporation, College Station, Texas, USA). Results are reported as odds ratios and $95 \%$ confidence intervals.

\section{RESULTS}

Data on of 3578 patients were abstracted. The Carstairs values ranged from -5.26 (least deprivation) to +8.38 (most deprivation). Table 1 presents demographic data including the median value for each quintile and preoperative variables. Patients in the highest quintiles waited on average 14 days longer for surgery than patients in the lowest quintiles, but this did not reach significance (table 1). High deprivation scores were associated with younger age, greater body mass index, diabetes, smoking at time of surgery, and higher EuroSCOREs (table 1). No differences were observed across the quintiles with respect to the extent of coronary artery disease or operative priority.

Table 2 presents perioperative variables. Socioeconomic deprivation was associated with increased inotrope use, with lower values being found in the highest quintiles $(p=0.004)$ (table 2 ). Thirty day mortality ranged from $1.2-1.4 \%$ and no significant trend was found across the quintiles. There were, however, more postoperative myocardial infarctions in the most deprived groups (1.8\% of patients in quintile 1 and $3.3 \%$ of patients in quintile $5 ; p=0.05)$. Hospital length of stay was also significantly longer in the most deprived groups $(p=0.03)$. After adjustment for EuroSCORE (which already includes independent risk factors such as age and sex), significant associations between socioeconomic deprivation and postoperative myocardial infarction and combined outcome of 30 day mortality, postoperative myocardial infarction, and stroke were found (table 3).

\section{DISCUSSION}

To the best of our knowledge, this is the first study to show that, in a British population undergoing $C A B G$, socioeconomic deprivation is an independent predictor of poor cardiovascular outcome. This finding is important because postoperative myocardial infarction has been shown to adversely affect late prognosis. ${ }^{17}$

Several explanations may address our findings. The first of these relates to comorbidity. Salomaa and colleagues ${ }^{18}$ suggested that diseases other than coronary artery disease may accumulate among people with low socioeconomic status and influence their prognosis after myocardial infarction. The most deprived patients in our study did present with a significantly higher incidence of baseline risk factors including body mass index, diabetes mellitus, and smoking at time of surgery. The American Heart Association recognises obesity as a major risk for coronary artery disease, ${ }^{19}$ and the Society of Thoracic Surgeons database reported that morbid obesity is an independent predictor of increased operative mortality in patients undergoing $\mathrm{CABG} .{ }^{20}$ In addition, obesity has been independently associated with coronary endothelial dysfunction even in patients with normal or mildly diseased coronary arteries. ${ }^{21}$ Smoking has been strongly associated with myocardial infarction, particularly in women, ${ }^{22}$ and several studies have shown the association between diabetes and coronary artery disease. ${ }^{19}$

Secondly, differences in psychosocial factors may provide an additional explanation. In BHAT ( $\beta$ blocker heart attack trial) patients classified as being socially isolated and having a high degree of life stress had more than a four times greater risk of late death after myocardial infarction than men with low levels of both stress and isolation..$^{23}$ The role of psychosocial risk factors and non-fatal myocardial infarction has been highlighted, ${ }^{24}$ as has the possible association between type A personality and risk of non-fatal myocardial infarction mediated by alterations in high density lipoprotein cholesterol concentration. $^{25}$

Thirdly, differences in the incidence of perioperative myocardial infarction may also be caused by differences in 
treatment and in those seeking treatment. It has recently been reported that medications with proven effect in secondary prevention, such as $\beta$ blockers, antiplatelet agents, and hypolipidaemic agents, were less often prescribed after myocardial infarction for men with low income than for men with high income. $^{1826}$

We were unable to confirm the results of a recent Italian study designed to measure socioeconomic inequalities in access to CABG and 30 day mortality in Rome. The authors found a significant association between social class and mortality after surgery, ${ }^{27}$ but this may be explained by the following. Firstly, the cohort was older than the one we observed. Secondly, social class was used as an index to measure socioeconomic status, which included items of level of education. This may be a more sensitive method than measuring area deprivation. Thirdly, there are many differences in risk factors and incidence of coronary artery disease between Mediterranean and British populations. ${ }^{28}$ Lastly, although our findings suggest that poor cardiovascular outcomes following CABG may be affected by socioeconomic deprivation, our study was underpowered to detect differences in 30 day mortality, although observed trends suggested that both mortality and stroke were higher in the most adverse quintile of deprivation score. We found no evidence of a graded "dose-response" relation, but it seems more likely that the effects of socioeconomic deprivation as measured by an area deprivation score may be insensitive to more moderate levels of deprivation compared with individual socioeconomic status. As no data were available on occupation, educational attainment, or material wealth it was not possible to examine this further.

Some limitations in our study design need to be mentioned. Firstly, ethnicity has also been associated with socioeconomic deprivation, ${ }^{89}$ but in our cohort this was a factor in only $1 \%$ of the population. We therefore did not include this variable in our analysis. Secondly, less deprived patients tend to opt for CABG privately. It has been estimated that private sector activity for coronary revascularisation may add another $10-20 \%$ above the National Health Service (NHS) rates. ${ }^{30}$ However, on the basis of the results of this study, it seems reasonable to suggest that the inclusion of those patients should increase the observed differences.

Current health policy is committed to reducing health inequalities and in particular improving access to specialist services. ${ }^{31}$ Access to high quality specialist services is recognised as an important determinant of health status and it has been suggested that policies designed to provide equal access to acute health services may be cost effective in addressing the consequences of deprivation. ${ }^{32}$ Regarding coronary artery disease, providing additional resources and cardiac procedures has been shown to increase the revascularisation rates across all socioeconomic groups, ${ }^{33}$ but data on the improvement of clinical outcome with this policy has yet to be presented. It has been argued that targeting aspects of poor health among society's poorer groups may also address the associations found between socioeconomic deprivation and poor health. ${ }^{32}$ However, this depends on a political commitment to reducing socioeconomic inequalities and on using a complex combination of strategies often involving a multidisciplinary approach. ${ }^{32}$

In conclusion, our study suggests that patients undergoing $\mathrm{CABG}$ living in areas with high deprivation scores are younger, have more clinical risk factors, and experience more postoperative cardiovascular complications than patients living in low deprivation score areas.

\section{ACKNOWLEDGEMENTS}

We thank Dr Y Ben Shlomo and Professor Shah Ebrahim for their helpful comments regarding this manuscript.

\section{Authors' affiliations}

F C Taylor, R Ascione, P Narayan, G D Angelini, Bristol Heart

Institute, University of Bristol, Bristol, UK

K Rees, Department of Social Medicine, University of Bristol

F C Taylor and R Ascione contributed equally to this study and are co-principal investigators.

\section{REFERENCES}

1 Ben Shlomo Y, Chaturvedi N. Assessing equity in access to health care provision in the UK: does where you live affect your chances of getting a coronary artery bypass graft. J Epidemiol Commun Health 1995;49:200-4.

2 Hemingway $\mathbf{H}$, Shipley M, MacFarlane $P$, et al. Impact of socioeconomic status on coronary mortality in people with symptoms, electrocardiaographic abnormalities, both or neither: the original Whitehall study 25 year follow up. J Epidemiol Commun Health 2000;54:510-6.

3 Alter D, Naylor D, Austin P, et al. Effects of socioeconomic status on access to invasive cardiac procedures and on mortality after acute myocardial infarction. N Engl J Med 1999;341:1359-67.

4 Manson-Siddle C, Robinson M. Does increased investment in coronary angiography and revascularisation reduce socioeconomic inequalities in utilisation. J Epidemiol Commun Health 1999;53:572-7.

5 Black N, Langham S, Petticrew M. Coronary revascularisation: why do rates vary geographically in the UK? J Epidemiol Commun Health 1995;49:408-12

6 Kaplan G, Keil J. Socioeconomic factors and cardiovascular disease: a review of the literature. Circulation 1993;88:1973-98.

7 McLeod M, Finalyson A, Pell J, et al. Geographic, demographic and socioeconomic variations in the investigation and management of coronary heart disease in Scotland. Heart 1999;81:252-6

8 Philbin E, McCullogh P, DiSalvo T, et al. Socioeconomic status is an important determinant of the use of invasive procedures after acute myocardial infarction in New York State. Circulation 2000;102:III10715.

9 Taylor F, Angelini G, Victory J. Up-take of cardiac rehabilitation among patients following cardiac bypass surgery. Heart 2001:86:92-3.

10 Michelozzi $\mathbf{P}$, Perucci $C$, Forastiere $F$, et al. Inequality in health: socioeconomic differentials in mortality in Rome, 1990-95. J Epidemiol Commun Health 1999;53:687-93

11 Pell J, Pell A, Norrie J, et al. Effect of socioeconomic deprivation on waiting time for cardiac surgery: a retrospective cohort study. BM 2000;320: 15-9.

12 Sculpher M, Petticrew M, Kelland J, et al. Resource allocation for chronic stable angina: a systematic review of effectiveness, costs and cost-effectiveness of alternative interventions. Health Technology Assessment 1998;2(10).

13 Lytle BW, Blackstone EH, Loop FD, et al. Two internal thoracic artery grafts are better than one. J Thorac Cardiovasc Surg 1999;1 17:855-72.

14 Nashef S, Roques F, Michel P, et al. European system for cardiac risk evaluation (EuroSCORE). Eur J Cardiothorac Surg 1999;16:9-13.

15 Carstairs V, Morris R. Deprivation and health in Scotland. Aberdeen:Aberdeen University Press, 1991.

16 Ascione R, Caputo M, Calori G, et al. Predictors of atrial fibrillation after conventional and beating heart coronary surgery: a prospective, randomized study. Circulation 2000;102:1530-5.

17 Force T, Hibberd P, Weeks G, et al. Perioperative myocardial infarction after coronary artery bypass surgery: clinical significance and approach to risk stratification. Circulation 1990;82:903-12

18 Salomaa V, Miettinen $\mathrm{H}$, Niemela M, et al. Relation of socio-economic position to the case fatality, prognosis and treatment of myocardial infarction events: the FINMONICA MI register study. J Epidemiol Commun Health 2001;55:475-82.

19 St Jeor ST, Brownell KD, Atkinson RL, et al. Obesity. Workshop III. AHA prevention conference III. Behavior change and compliance: keys to improving cardiovascular health. Circulation 1993:88:1391-6.

20 Edwards FH, Carey JS, Grover FL, et al. Impact of gender on coronary bypass operative mortality. Ann Thorac Surg 1998;66:125-31.

21 Al Suwaidi J, Higano ST, Holmes DR, et al. Obesity is independently associated with coronary endothelial dysfunction in patients with normal or mildly diseased coronary arteries. J Am Coll Cardiol 2001;37:1523-8.

22 Niolstad I, Arnesen E, Lund-Larsen PG. Smoking, serum lipids, blood pressure, and sex differences in myocardial infarction. Circulation 1996;93:450-6.

23 Ruberman W, Weinblatt E, Goldberg JD, et al. Psychosocial influences on mortality after myocardial infarction. $N$ Engl J Med 1984;311:522-59.

$24 \mathrm{O}^{\prime}$ Connor NJ, Manson JE, O'Connor GT, et al. Psychosocial risk factors and nonfatal myocardial infarction. Circulation 1995;92:1458-64.

25 Friedman M, Rosenman RH. Association of specific overt behaviour pattern with blood and cardiovascular findings. JAMA 1959;169: 1286-96.

26 Rathore SS, Berger AK, Weinfurt KP, et al. Race, sex, poverty, and the medical treatment of acute myocardial infarction in the elderly. Circulation 2000;102:642-8. 
27 Ancona C, Agabiti N, Forastiere F, et al. Coronary artery bypass graft surgery: socioeconomic inequalities in access and in 30 day mortality. A population-based study in Rome, Italy. J Epidemiol Commun Health 2000;54:930-5.

28 De Logeril M, Salen P, Martin J, et al. Mediterranean diet, traditional risk factors and the rate of cardiovascular complications after myocardial infarction. Circulation 1999;99:779-85

29 Franks A, May D, Wenger N, et al. Racial differences in the use of invasive coronary procedures after acute myocardial infarction in medicare beneficiaries. Ethn Dis 1993;3:213-20.
30 Payne N, Saul C. Variations in use of cardiology services in a health authority: comparison of coronary artery revascularisation rates with prevalence of angina and coronary mortality. BM 1997;314:257-61.

31 Anon. National Service Framework. Coronary heart disease. Department of Health, 2000

32 Arblaster $L$, Lambert $M$, Entwhistle $V$, et al. A systematic review of the effectiveness of health service interventions aimed at reducing inequalities in health. J Health Serv Res Policy 1996;1:93-103.

33 Manson-Siddle C, Robinson M. Super profile analysis of socioeconomic variations in coronary investigation and revascularisation rates. $J$ Epidemiol Commun Health 1998;52:507-1.

\section{IMAGES IN CARDIOLOGY}

\section{Rheumatic carditis in an 18 year old man}

$A^{\prime \prime}$ 18 year old man presented with progressive dyspnoea for three months. Seven months before admission he had suffered from choreatic movements of the right arm. A month before admission the general practitioner treated him for diagnosed pericarditis with high dosages of salicylates.

At physical examination the patient appeared chronically ill and in biventricular congestive cardiac failure, with findings of mild aortic regurgitation (AR), severe mitral (MR) and tricuspid regurgitation (TR), in the presence of a dilated left ventricle. The chest $x$ ray showed cardiomegaly with pronounced left atrial dilatation (below, upper panel).The 12 lead ECG showed a sinus tachycardia, first degree atrioventricular conduction delay, and left atrial dilatation, while rhythm monitoring showed intermittent second degree type II atrioventricular conduction delay (below, lower panel). Laboratory assessment revealed a raised $C$ reactive protein, erythrocyte sedimentation rate, anti-streptolysin O titre, and anti-DNAse B. The culture of the throat swab remained negative. Transthoracic echocardiography (right, upper panel) confirmed mild AR, and severe MR and TR with a mildly dilated left ventricle and preserved systolic function. The right ventricular pressures were raised $(50 \mathrm{~mm} \mathrm{Hg})$. The clinical
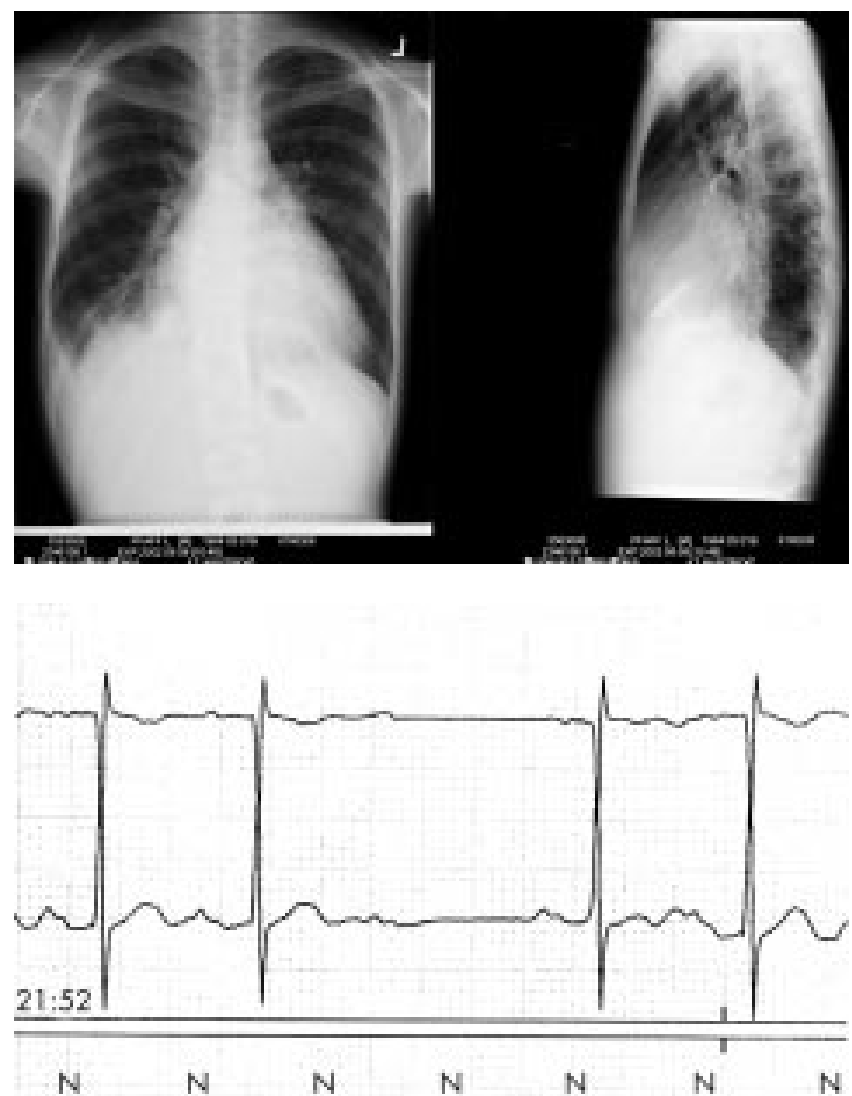

diagnosis of rheumatic carditis was considered in the presence of two major and two minor revised Jones criteria. Since thiscondition is rarely diagnosed in the Netherlands, and diagnostic uncertainty remained, it was decided to obtain left ventricular myocardial biopsies. In two of the three samples Aschoff bodies, pathognomonic for rheumatic carditis, were found (right, lower panel).

The patient was treated with penicillin, loop diuretics, angiotensin converting enzyme inhibitors, digitalis, and $\beta$ blockers and his condition improved significantly.

$R$ van Kimmenade J P Smedema R J van Suylen rki@cardio.azm.nl
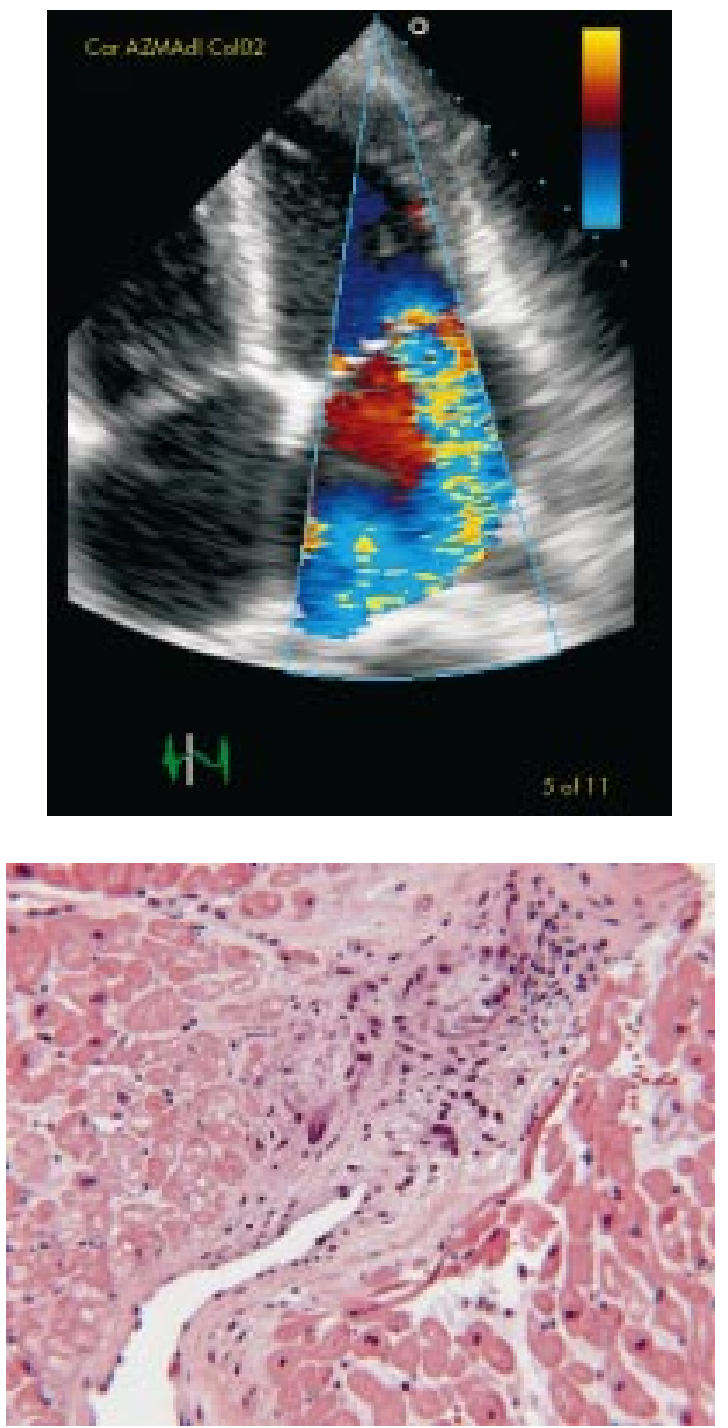